\title{
Memory span for heterospecific individuals' odors in an ant, Cataglyphis cursor
}

\author{
EMmeline Foubert ANd Elise Nowbahari \\ LEEC CNRS UMR 7153, Université Paris 13, Villetaneuse, France
}

\begin{abstract}
Only recently have researchers studied the ability of ants to learn and remember individual heterospecific odors. Cataglyphis cursor adults have the capacity to learn these odors, but the duration of their memory and the factors that affect its formation remain unknown. We used a habituation/discrimination paradigm to study some of these issues. C. cursor adult workers were familiarized to an anesthetized Camponotus aethiops on four successive encounters. Then they were either isolated or placed with 20 nestmates for a certain length of time before undergoing a discrimination test that consisted of reintroducing the familiar $C$. aethiops, as well as introducing an unknown member of the same colony. The results showed that adult $C$. cursor ants can retain in memory a complex individual odor for at least $30 \mathrm{~min}$, as well as differentiate it from the odor of another closely related individual. However, when ants were replaced in a rich social background between the habituation and the discrimination trials, we did not observe a significant discrimination between the known and unknown C. aethiops ants. Our study shows, for the first time, the existence of long-term memory for individual odors in mature ant workers.
\end{abstract}

Learning abilities in insects are well documented, especially for navigation and foraging activities. Bees and ants are able to learn visual pattern sequences and to reuse them to orient themselves (Chameron, Schatz, PastergueRuiz, Beugnon, \& Collett, 1998; Horridge, 2006; Macquart \& Beugnon, 2004; Zhang, Mizutani, \& Srinivasan, 2000 ). Bees are also well known for their notable olfactory learning and memory abilities (Horridge, 2006; Zhang et al., 2000). These olfactory abilities have been studied in other models such as fruit flies (Drosophila melanogaster; Davis, 2005), crickets (Gryllus bimaculatus; Matsumoto \& Mizunami, 2000, 2005), and cockroaches (Periplaneta americana; Sakura \& Mizunami, 2001; Sakura, Okada, \& Mizunami, 2002; Watanabe, Kobayashi, Sakura, Matsumoto, \& Mizunami, 2003).

Among social insects, olfactory abilities are essential in kin and colonial recognition. In a colony, every individual carries a "gestalt odor" across the surface of the body. This mixture comprises the odors of all of the colony's individuals and is spread through trophallaxis and licking (Errard, Hefetz, \& Jaisson, 2006; Lahav, Soroker, Hefetz, \& Vander Meer, 1999; Lenoir, Fresneau, Errard, \& Hefetz, 1999). Early in adult life, each colony member must learn these cues, which, when encoded as a template, serve not only to determine the colonial membership of other individual ants, but also to discriminate among them (Crozier \& Pamilo, 1996).

The learning of colonial odor in Cataglyphis cursor takes place during the first larval stage (Isingrini, Lenoir, \& Jaisson, 1985). This learning is predicted to be stable; information acquired during the larval stage is known to persist through the metamorphosis into adulthood. However, there is another learning period after adult emergence (Isingrini et al., 1985; Jaisson, 1974). Moreover, the colonial visa is flexible because it depends on each individual odor, the colony's demographic fluctuations (Breed \& Bennett, 1987), the season, and resources (Nielsen, Boomsma, Oldham, Petersen, \& Morgan, 1999; Provost, Bonavita-Cougourdan, \& Rivière, 1994; Vander Meer \& Morel, 1998; Vander Meer, Saliwanchik, \& Lavine, 1989). That is why the template must be constantly updated and requires cerebral plasticity - in particular, efficient learning and memory abilities.

Crozier (1987) proposed another model for both primitive ant societies and other insect societies that have relatively few individuals. In this model, there is no gestalt odor process; each individual keeps its own chemical characteristics. Recognition of each of the members of the colony occurs via individual recognition. In typical ant societies, in which individuals live in colonies of hundreds or thousands of individuals, this kind of recognition system was assumed to be improbable. However, contrary to this assumption, recent studies have shown homospecific individual discrimination and recognition among social insects, such as wasps (Polistes fuscatus; Tibbetts, 2002) and founding queen ants (Pachycondyla villosa; D'Ettore \& Heinze, 2005). Both systems are characterized by long-term, stable dominance hierarchies enforced by individual aggression.

Among Cataglyphis ants, discrimination of heterospecific individuals and nonnestmate homospecific individuals occurs in a nonhierarchical context, and the learning of each individual odor was observed using a habituation 
process without reinforcement (Nowbahari, 2007). These heterospecific and homospecific individual discrimination abilities in workers can be understood in terms of the "dear enemy" phenomenon with regard to allocolonial or heterospecific individuals and in the context of the preference networks among members of the same colony (Delattre \& Nowbahari, 2007). Individual recognition in a hierarchical context seems to require long-term memory and is robust enough not to be erased by several encounters (Dreier, van Zweden, \& D'Ettorre, 2007). In this study, we tested whether the memory of the odor of a worker ant from a different species (i.e., Camponotus aethiops) would be retained by C. cursor ants in long-term memory, as well as whether this learning would be imperturbable.

\section{GENERAL METHOD}

\section{Ants and Rearing Conditions}

Two ant species were used for these experiments: C. cursor and C. aethiops. For each test series, we tested 16 C. cursor from four monogynous colonies, each of which included one reproductive queen. Three of these colonies were sampled at Menerbes, and the fourth was sampled at Bonnieux (Vaucluse, France) in April 2006. These colonies were reared in the laboratory in a cylindrical, closed nest connected with a foraging area. Ants were fed on mealworm larvae and apple-honey mixture twice a week. The temperature of the breeding room was kept at $28^{\circ} \pm 2^{\circ} \mathrm{C}$, with a humidity level of $20 \%$ to $40 \%$ and a $12: 12$-h light:dark cycle.

C. aethiops ants were used as stimuli. This species is sympatric with $C$. cursor. The two colonies used for these experiments were collected in Touraine, France, in 2006, and were reared in the same laboratory, in the same room, and under the same conditions as the C. cursor colonies.

\section{Procedure}

We used the habituation/discrimination test method usually applied in studies of vertebrates (see, e.g., Todrank \& Heth, 2003) and adapted for use with ants (Nowbahari, 2007). For each experimental series, 16 C. cursor workers (4 from each colony) were removed from the foraging area near the nest entrance and individually marked on the abdomen by a distinct spot of odorless, indelible paint (Uni Paint Marker PX 20, Mitsubishi Pencil Co.). The ants were placed in a box with other test members from their colony, applehoney mixture, and moisturized cotton, until the beginning of the tests the following day.

Encounters occurred in a circular box that was $3.5 \mathrm{~cm}$ in diameter. Two individuals were tested in parallel for the purpose of making crossed encounters (Figure 1). C. cursor workers were set in the encounter area at least $1 \mathrm{~min}$ before the presentation of the stimulus in order to reduce their excitability following the manipulation. The test area surface was covered with filter paper and changed after each encounter to avoid chemical markings. During the $10 \mathrm{~min}$ between encounters, the ant was gently placed in an individual box with wet cotton, allowing it to drink. Stimulus $C$. aethiops were anesthetized with $\mathrm{CO}_{2}$ to prevent their behavior from biasing the response of $C$. cursor. The stimulus ants were kept under an anesthetic between encounters. This procedure (using $\mathrm{CO}_{2}$ ) allowed us to keep stimulus ants alive and avoid chemical alterations that would induce behavioral modifications (necrophoric behavior) in individuals perceiving it (Ataya \& Lenoir, 1984; Wilson, Durlach, $\&$ Roth, 1958). $\mathrm{CO}_{2}$ anesthesia is more reliable than cooling, which can lead to substantial mortality rates. Moreover, the immobility duration acheived by cooling is shorter than that achieved by using $\mathrm{CO}_{2}$ anesthesia. Cold also restricts molecular volatility from the cuticle. In both experiments, stimuli ants (C. aethiops) were placed in a box with their nestmates during the rest period.

Two experiments were conducted. In Experiment 1, we tested the memory span for learned odors belonging to heterospecific individuals. Experiment 2 was carried out to determine whether the social exposure with the nestmate group would interfere with the heterospecific memory. In addition, a control test was carried out to ensure that olfactory marks had not been deposited on the stimulus ant.

All encounters were videotaped, and the occurrence of behavior patterns listed in Table 1 was blind counted. The sum of these occurrences determined the number of agonistic behaviors, which were analyzed using nonparametric inference with permutation tests (also called randomization tests) for paired samples and for independent

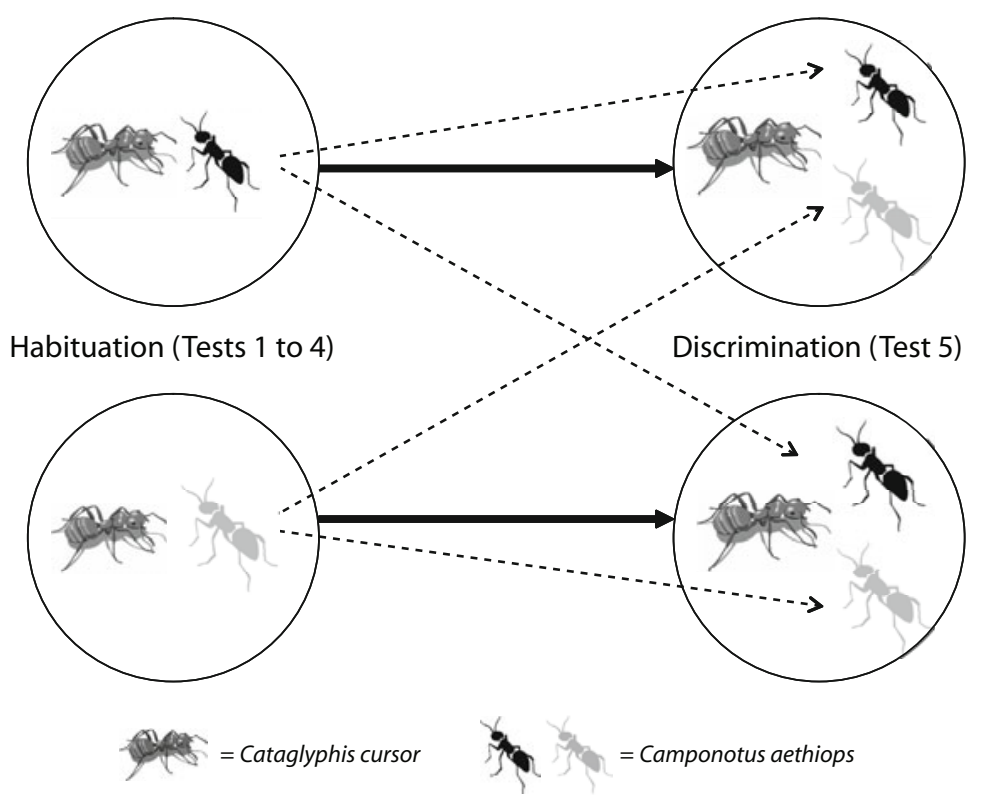

Figure 1. Schematic setup of the habituation/discrimination experimental procedure. 
Table 1

Observed Behaviors During Trials

\begin{tabular}{ll}
\hline \multicolumn{1}{c}{ Behavior } & \multicolumn{1}{c}{ Description } \\
\hline Opening of mandibles & $\begin{array}{l}\text { Opening of mandibles near the stimulus, } \\
\text { often after an antennal contact } \\
\text { Biting }\end{array}$ \\
Geizing the stimulus body with mandibles \\
& $\begin{array}{l}\text { Folding abdomen toward the stimulus, } \\
\text { frequently seizing it with mandibles, and } \\
\text { spraying formic acid }\end{array}$ \\
\hline
\end{tabular}

samples with StatXact 7 (Cytel, 2005). The statistics were considered significant at $p \leq .05$.

\section{EXPERIMENT 1 \\ Memory Span for \\ Heterospecific Individuals' Odors}

\begin{abstract}
Method
In this experiment, we tested the memory span for the learned odors belonging to heterospecific individuals. Each C. cursor worker was habituated to a C. aethiops anesthetized by $\mathrm{CO}_{2}$, in four successive encounters of $3 \mathrm{~min}$ each, separated by 10 -min intervals (habituation trials). After the four habituation trials, C. cursor ants were isolated from C. aethiops stimuli for a 10-, 30-, or 60-min interval (rest period). During this rest period, C. cursor ants were socially isolated. After the rest/isolation period, we proceeded with a discrimination test: For each C. cursor, we presented both the familiar and a homocolonial unknown C. aethiops.
\end{abstract}

\section{Results}

Habituation trials. During the four successive encounters, we observed habituation on agonistic behavior. C. cursor adult ants were more aggressive toward the stimulus $C$. aethiops during the first encounter than during the fourth (permutation test for paired samples significant in each situation: For 10 min of isolation, $n=16, p=$ .03 ; for $30 \mathrm{~min}$ of isolation, $n=15, p=.0009$; For these two situations together, $n=31, p=.0003$; for $60 \mathrm{~min}$ of isolation, $n=14, p=.04$ ) (Figure 2).

Discrimination test. When the rest period was either 10 min (a time period that was identical to that between the habituation trials) or $30 \mathrm{~min}$ in duration, C. cursor ants were able to discriminate between familiar and unfamiliar C. aethiops. More agonistic behaviors were exhibited toward the unfamiliar ant than toward the familiar one (permutation test for paired samples: For $10 \min , n=16, p=$ .0056 ; for $30 \min , n=15, p=.029$ ) (Figure 2). The sum of occurrences of agonistic behaviors of $C$. cursor toward the 2 stimulus $C$. aethiops was not significantly different during the discrimination test when we compared the 10 -min rest period test with the 30 -min rest period test (permutation test for two independent samples, $p=.80$ ).

However, when the rest period was $60 \mathrm{~min}, C$. cursor ants did not respond differently to the familiar and unfamiliar $C$. aethiops ants ( $n=14, p=.34$; Figure 2$)$.

\section{Discussion}

Our results from Experiment 1 showed that C. cursor adult ants are able to learn the individual odor of a heterospecific ant and discriminate it from the odor of an unfamiliar individual after at least $30 \mathrm{~min}$, which is considered long-term memory for an insect. The rare studies of long-term memory using habituation and stimuli that possess a particular social valence include those from our laboratory, using Cataglyphis niger (Nowbahari, 2007), and a recent study by Dreier et al. (2007), which showed that unrelated founding queens of $P$. villosa and Pachychondyla inversa retain information about the individual identities of other founding queens as long as $24 \mathrm{~h}$ after separation. However, Dreier et al. focused on a homospecific hierarchical context, in which individual recognition facilitates a stable linear dominance hierarchy between queens and workers. In this small-group context, the importance of individual recognition is obvious.

\section{EXPERIMENT 2 \\ The Effect of Social Environment on Lifetime Retention of Individual Learned Odors}

\section{Method}

This experiment was conducted to determine whether the social environment influenced the memory span. We proceeded as in Experiment 1, except that during the 10- or 30-min rest period, which followed immediately after the four habituation trials, each subject was placed in a small homocolonial group of 20 individuals taken directly from the nest.

\section{Results}

Habituation trials. As in Experiment 1, we observed habituation to the encountered C. aethiops. C. cursor ants were more aggressive against the stimulus $C$. aethiops during the first encounter than during the fourth (permutation test for paired samples: for the 10-min rest period, $n=14$, $p=.02$; for the 30 -min rest period, $n=14, p=.05$; for the two delays together, $n=28, p=.0016$ ) (Figure 3).

Discrimination test. Unlike the behavior we observed in Experiment 1, C. cursor ants did not show a significant discrimination between the $2 C$. aethiops stimulus ants, either after a $10-\min (n=14, p=.30)$ or $30-\min (n=14$, $p=.19$ ) rest period (see Figure 3). Although a comparison of the data in Experiments 1 and 2 might suggest that ants were simply less aggressive in Experiment 2, a statistical analysis of the interaction between the two experiments and the test stimuli revealed no differences in aggressive behavior between the two experiments (permutation test for two independent samples: for the 10-min rest period, $p=.18$; for the 30-min rest period, $p=.30$; for these two delays together, $p=.092$ ). As in Experiment 1, the occurrence of agonistic behavior of $C$. cursor toward 2 stimulus $C$. aethi ops was not significantly different when we compared the 10-min rest period test with the 30-min rest period test (permutation test for two independent samples, $p=.92$ ). In the same way, the sum of these occurrences was not significantly different when we compared Experiments 1 and 2 (permutation test for two independent samples, $p=.30$ ).

As in Experiment 1, the sum of occurrences of agonistic behaviors of $C$. cursor toward 2 stimulus $C$. aethiops was not significantly different when we compared the 10-min rest period test with the 30-min rest period test (permutation test for two independent samples, $p=.92$ ). In the 


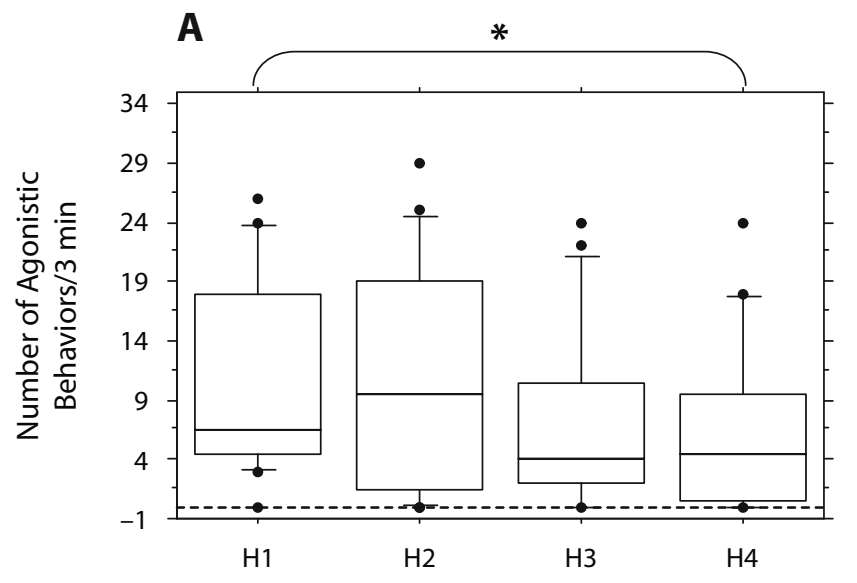

Habituation 10-min Isolated
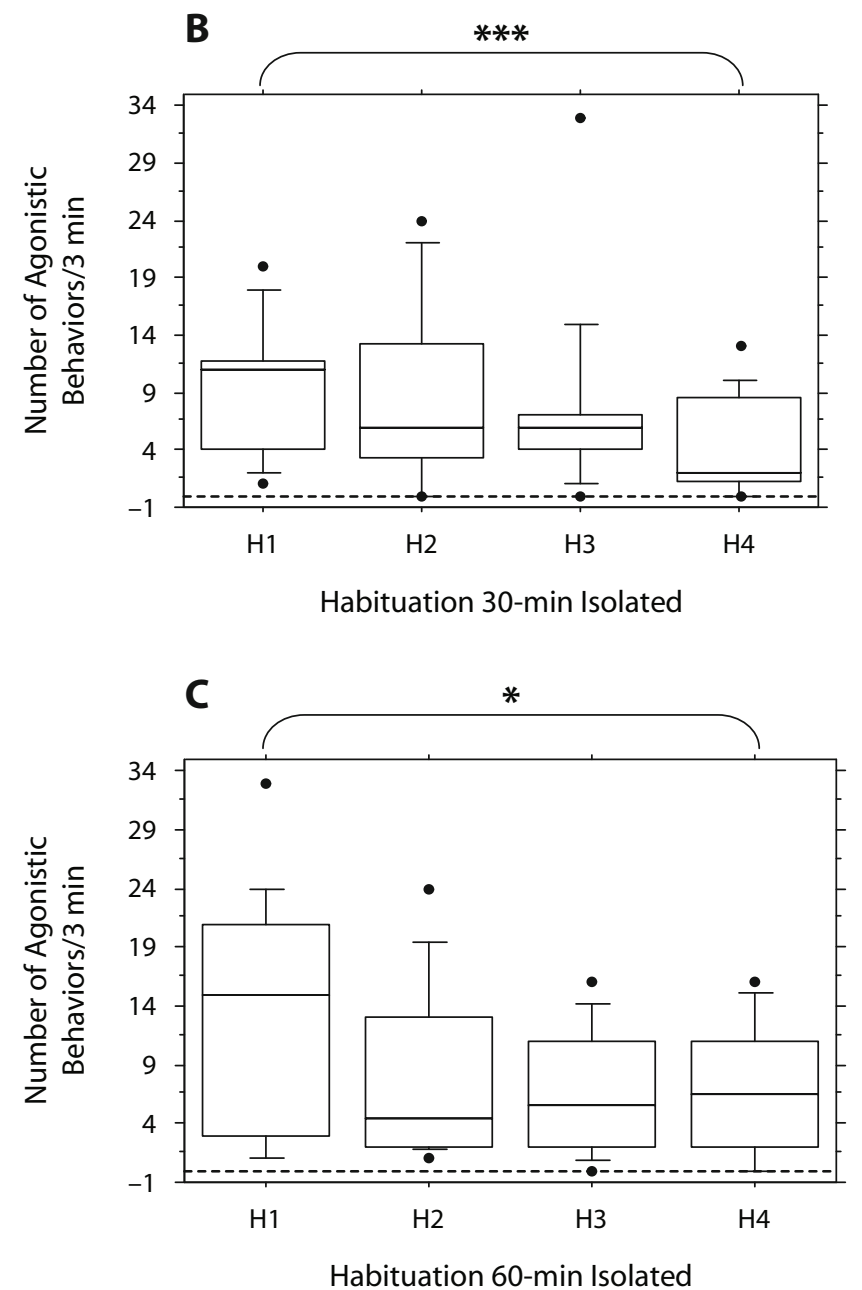

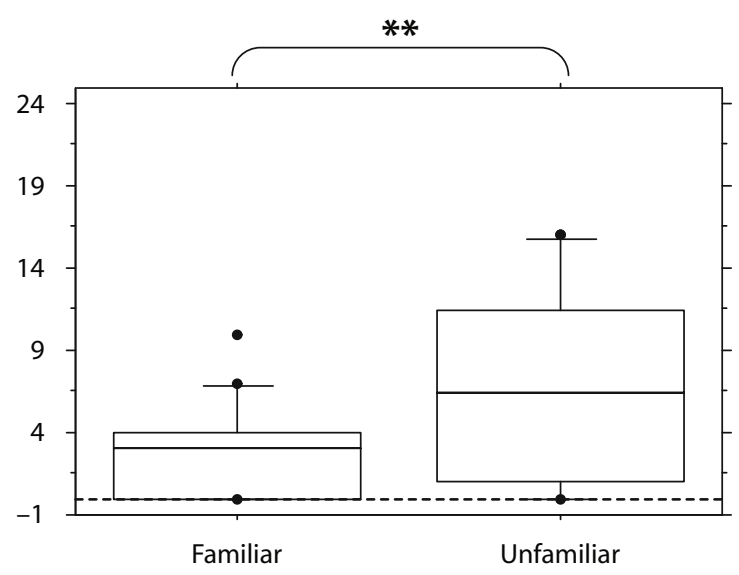

Discrimination 10-min Isolated

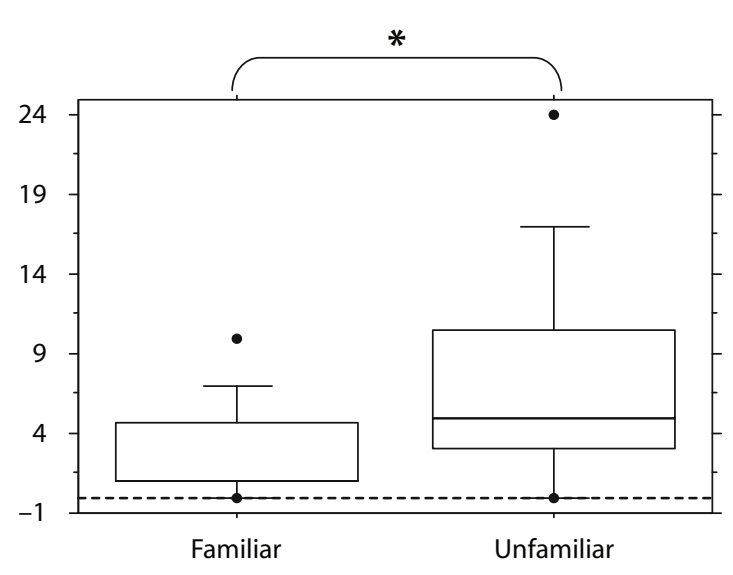

Discrimination 30-min Isolated

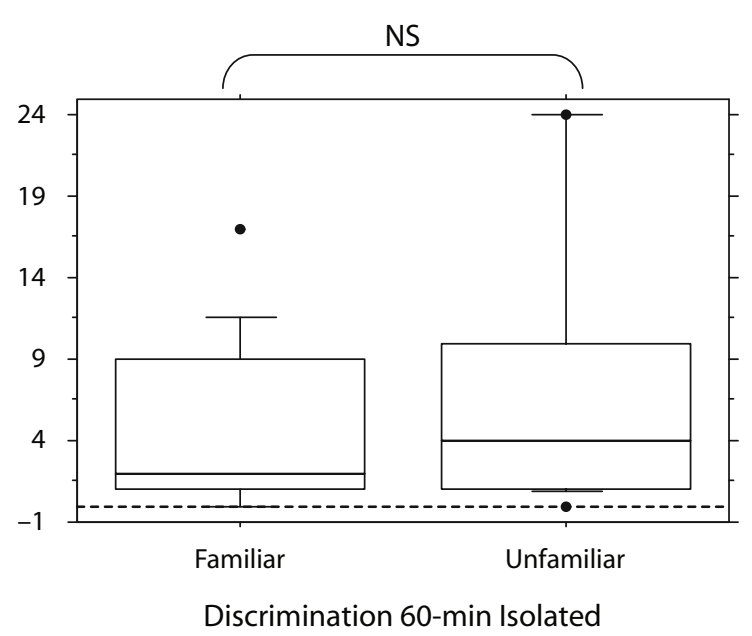

Figure 2. Number of agonistic behaviors expressed by a C. cursor ant toward a Camponotus ant in 3 min during the habituation/ discrimination procedure for three experimental conditions: (A) $10 \mathrm{~min}$, (B) $30 \mathrm{~min}$, or (C) $60 \mathrm{~min}$ of rest period, during which C. cursor ants were isolated. Horizontal lines represent the 10th, 25 th, 50 th (median), 75 th, and 90th percentiles. Scores above the 90 th and below the 10th percentiles are plotted as individual points. ${ }^{*} p \leq .05 .{ }^{* * *} p \leq .01 .{ }^{* * * *} p \leq .001$. 

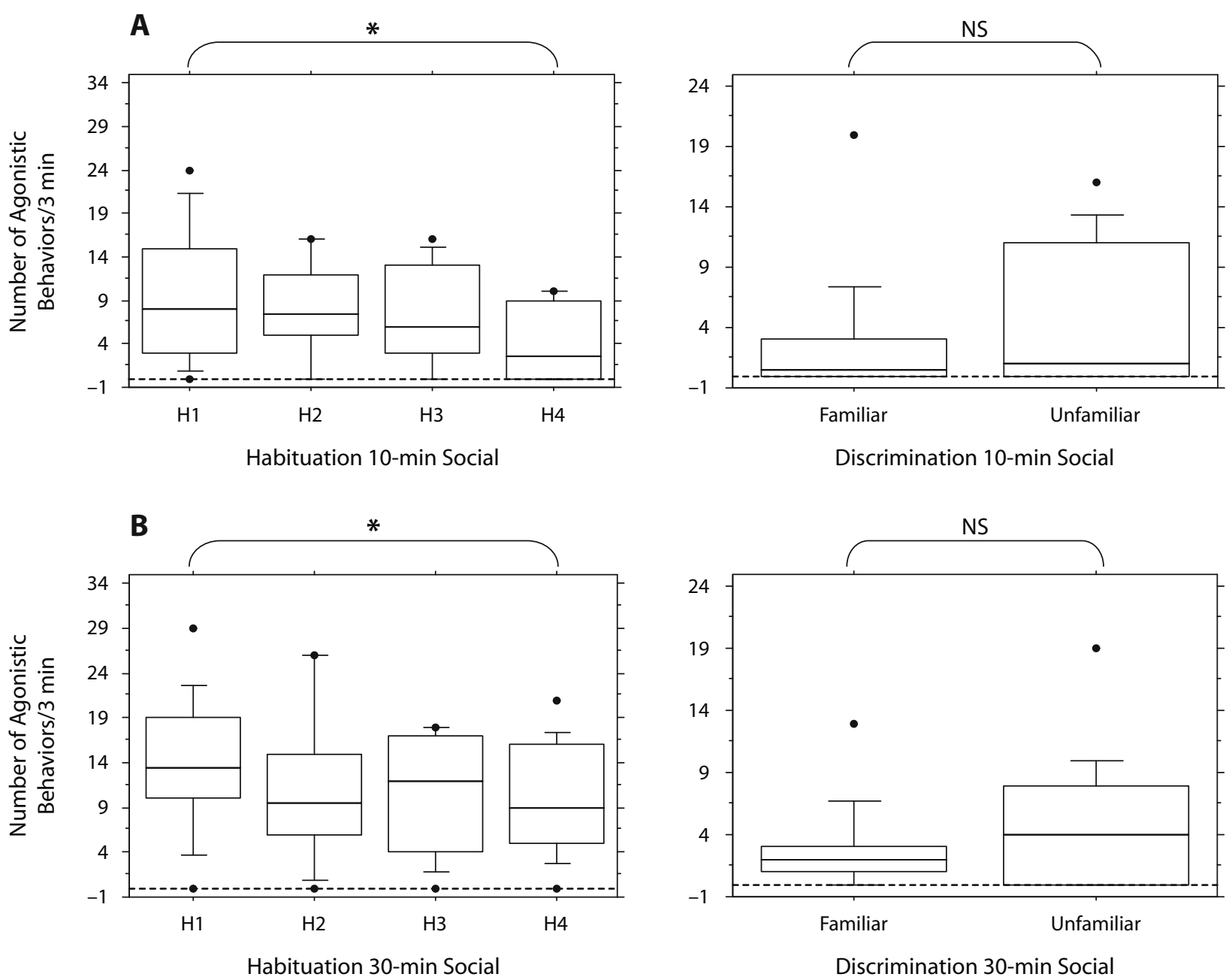

Figure 3. Number of agonistic behaviors expressed by a C. cursor ant toward a Camponotus ant in 3 min during the habituation/ discrimination procedure for a rest period of (A) 10 min and (B) 30 min, during which $C$. cursor ants were set back with 20 nestmates. Horizontal lines represent the 10th, 25th, 50th (median), 75th, and 90th percentiles. Scores above the 90th and below the 10th percentiles are plotted as individual points. ${ }^{*} p \leq .05$.

same way, the sum of these occurrences was not significantly different when we compared Experiments 1 and 2 (permutation test for two independent samples, $p=.30$ ).

\section{Discussion}

If C. cursor regains a rich social background of 20 nestmates after the habituation, as if returning to the nest, we descriptively observe a differentiation between two odor stimuli. The ants discriminate between the familiar and unfamiliar heterospecific individual odors, but the difference of intensity of agonistic behaviors toward unfamiliar individuals in comparison with that toward the familiar individual is less evident than in the individual condition (Experiment 1) and is nonsignificant. It seems that the ants were uncertain about how to choose and react toward known and unknown strangers. Simulation of a return to the nest may disturb access to memorized information, but it does not necessarily block it. That is, no certain evidence for memory loss is at hand. We propose tentatively that the ants did not actively delete "old" individual odor memories; rather, we suggest that the information may not have been recalled correctly, which is why the ant was not able to discriminate a familiar from an unfamiliar individual. This inhibitor effect may be due to the interference caused by the perception of its sisters' odors and/or by the update of the colonial template via contact with congeners. This effect is immediate, because $C$. cursor did not discriminate significantly between 2 C. aethiops ants after 10 min with its sisters.

Ichikawa and Sasaki (2003) showed, in honeybees, that the development of learning abilities requires social experience. Indeed, those abilities deteriorate when honeybees are socially deprived. Acquisition and maintenance of learning abilities require continual input of appropriate stimulation. The results of our study show that $C$. cursor ants are able to maintain the new individual information after being socially deprived, but returning to their nestmates perturbs or prevents individuals from discriminating 
perfectly between the two similar odors. However, Ichikawa and Sasaki studied social privation in young adults. In our study, we used mature individuals (older than 15 days).

The fact that $C$. cursor ants reintroduced into their social environment do not discriminate between two C. aethi ops does not mean that they cannot distinguish between the two individuals. Our statistical analysis shows that returning to the social environment does not decrease ants' aggressiveness, but causes a lapse of memory. Cheng and Wignall's (2006) experiments on honeybees showed how the learning of a second task interfered with what had been previously learned. Their results implicated response competition as a major contributor to the retroactive interference effect. The honeybees, like our C. cursor ants, seemed to hold on to memories of the learned task. In C. cursor, we suggest that returning to nestmates did not fully eliminate the ants' memory of learned odors.

\section{CONTROL EXPERIMENT Controlling for Olfactory Marking of C. aethiops Stimulus Ants}

\section{Method}

The control experiment was conducted to determine whether $C$. cursor deposited olfactory marks on the $C$. aethiops stimulus. To distinguish between two stimuli-a familiar individual and an unfamiliar individual — individuals may learn idiosyncratic particularities of the familiar one, or simply mark it, actively or not, with a recognition label. To control for this possibility, we used a procedure in which $C$. aethiops stimuli were substituted with a neutral stimulus.

We chose pellets of a fixative gum (UHU Patafix) as a neutral stimulus, not only because it has no alimentary or social valence (since it is a nonliving stimulus), but also because it causes aggressiveness in C. cursor ants. The only way for the ants to discriminate between two identical gum pellets would be to mark the familiar one with an olfactory label.

We proceeded as in Experiment 1, with a rest period of $10 \mathrm{~min}$. In each habituation trial, we presented a pellet of fixative gum to the $C$. cursor subject. In the discrimination test, we presented the familiar pellet and a clean, unfamiliar one that had been molded at the same time as the familiar one.

\section{Results}

Habituation trials. During four successive trials, as in Experiment 1, C. cursor ants exhibited agonistic behavior in the first encounter with fixative gum pellets, and it decreased during the four successive trials (permutation test for linked data, $n=20, p=.03$ ).

Discrimination test. No difference in agonistic behavior was observed between encounters with the familiar and the unfamiliar fixative gum pellets $(n=20, p=1$; Figure 4).

\section{Discussion}

The control experiment with fixative gum pellets suggests that C. cursor does not mark - that is, does not deposit a chemical substance on - the stimulus and does not, therefore, recognize it on subsequent encounters. Even though the tested ants decreased their agonistic behavior over successive encounters with the gum pellet (and thus habituated to this odor), they did not respond differently between the previously encountered pellet and another, novel pellet in the discrimination test. This absence of marking is not surprising: Marking could occur only through the deposition of alarm pheromones - which are very volatile - or through the deposition of cuticular hydrocarbons that are produced during allogrooming, which was absent here.

Ants readily discriminate between novel objects, particularly those possessing a novel color and odor, and show some reactions to them. In this experiment, for example, ants behaved aggressively toward the gum pellets, which were yellow and had a special odor. Indeed, the color and odor of the gum pellets were particularly effective in releasing aggressive behavior, even if the level of aggression was lower than that toward the anesthetized ants and decreased more rapidly. Moreover, the interpretation
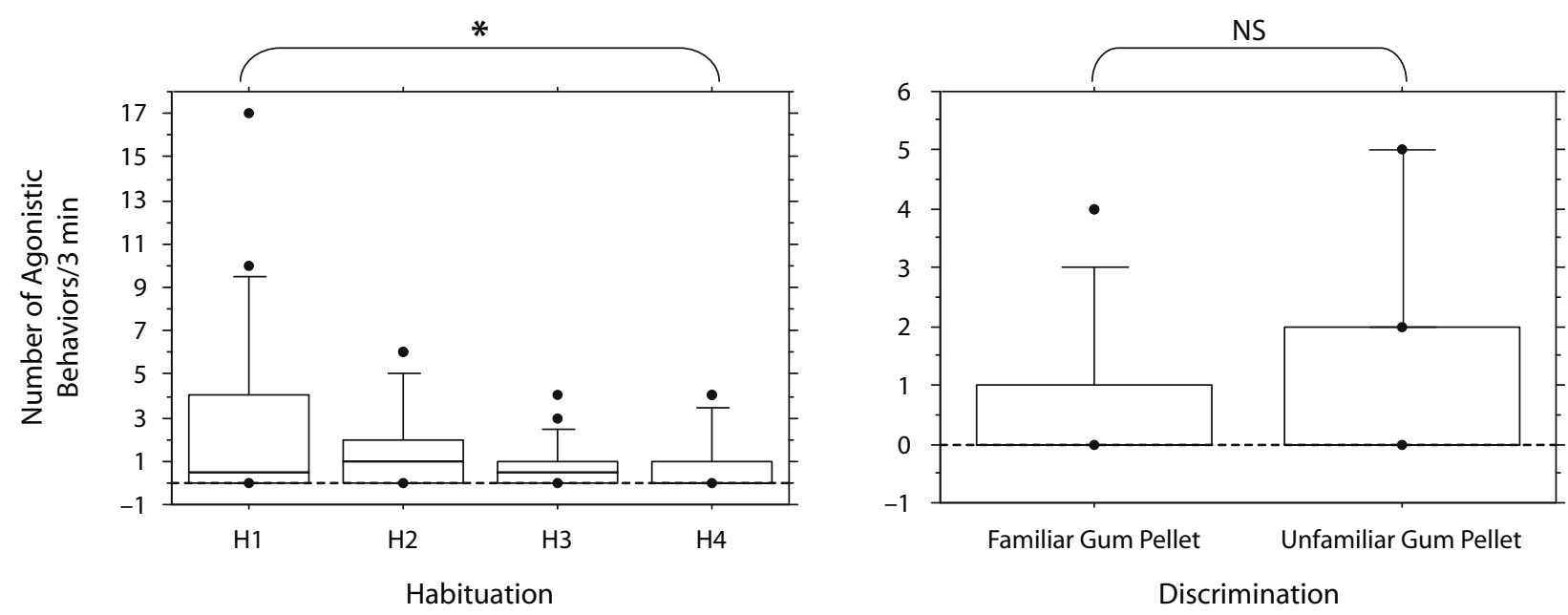

Figure 4. Number of agonistic behaviors expressed by a $C$. cursor ant toward a fixative gum pellet in 3 min during the habituation/ discrimination procedure with a rest period of $10 \mathrm{~min}$, during which the $C$. cursor ants were isolated. Horizontal lines represent the 10th, 25th, 50th (median), 75th, and 90th percentiles. Scores above the 90th and below the 10th percentiles are plotted as individual points. $\stackrel{*}{p} \leq .05$. 
that ants do not recognize individuals by marking them is supported by two additional arguments. First, because we employed a crossed design (see Figure 1), the unfamiliar C. aethiops ant used in the discrimination test would have been the same individual used in the habituation trials of a different $C$. cursor subject. Thus, if our $C$. cursor subjects had marked individuals with an odor-which would necessarily have been very similar, because the subjects were nestmates - then this mark should have interfered with the ants' ability to discriminate the familiar self-marked C. aethiops from the unfamiliar nestmate-marked stimulus ant. Second, unpublished data from our lab show that when anesthetized stimulus ants are returned to their own C. aethiops nestmates, those nestmates do not respond aggressively to them. If, in those experiments, a C. cursor subject ant had deposited any chemical marks on a C. aethiops stimulus ant, the stimulus ant would have been attacked immediately by her nestmates on her return to the nest.

Another way to discriminate the stimulus subjects without using mnemonic abilities would be by obtaining the stimuli's odorant cues, which they carry away with them and then use as a template. However, we excluded this possibility, because this does not require learning processes highlighted by the habituation phenomena.

\section{GENERAL DISCUSSION}

These results confirm and extend previous studies in Cataglyphis ants (Nowbahari, 2007) and show that C. cursor adult ants are able to learn a heterospecific individual's odor and discriminate it spontaneously, without reinforcement, from the odor of another close individual. We show here for the first time that the retention interval of the learned odor is at least $30 \mathrm{~min}$. After a 60 -min interval, however, C. cursor ants are not able to discriminate between the familiar and unfamiliar stimuli.

Dupuy, Sandoz, Giurfa, and Josens (2006) showed that two Camponotus species, C. mus and C. fellah, were able to learn simple odors (limonene and octanal, heptanal and 2-heptanone) in both positive and negative reinforcement tasks, using sucrose and quinine, respectively. Their tests demonstrated a retention time of at least $5 \mathrm{~min}$. Matsumoto and Mizunami (2000) showed that G. bimaculatus crickets have long-lasting olfactory learning abilities: They are able to remember a simple odor up to 7 days after three operant conditioning sessions. Others studies have demonstrated long-term memory abilities in other adult insects, such as honeybees, but those studies employed operant conditioning tasks using simple nutritive valence odorants (Hammer \& Menzel, 1995), whereas we used complex social odorants in a nonoperant conditioning task.

Our results complement the work of Dreier et al. (2007) by demonstrating analogous findings in a worker recognition abilities paradigm. In all other studies of heterospecific recognition (which has been explored extensively), the emphasis was placed on the role of cuticular hydrocarbons, especially in the early period of adult life. However, those studies focused on the imprint-like phenomenon of learning a global colonial odor, or gestalt odor learning, rather than individual discrimination ability. For example, Errard (1994) showed that when Formica selysi (Formicinae) and Manica rubida (Myrmicinae) ants were placed $5 \mathrm{~h}$ after emergence in a mixed heterospecific group, and then, after 3 months, separated and placed in homospecific groups, they recognized familiar heterospecific ants after up to 1 year of separation. Because cuticular hydrocarbon profiles have only traces of heterospecific hydrocarbons, self-reference is not a reliable recognition process (Errard, 1994). Learning that occurs shortly after emergence and is related to the colony - even an artificial colony - and to the nest is very stable. Our study shows that in mature ants, learning that occurs in the context of competitive interactions is stored for a shorter time in memory and is, therefore, more sensitive to external stimulation. Maintaining that information is possible only if the ant is likely to be confronted again with the same stimulus. In our study, we simulated an encounter in a foraging area with the forager ants. The probability of encountering the same heterospecific individual multiple times over a long time interval is low, even if we imagine that both individuals have overlapping foraging roads. Thus, it is not surprising to see the disappearance of individual discrimination of a familiar individual, especially after returning to the nest. In Errard's experiments, heterospecific individual recognition is linked to the mixed nature of the nest. Heterospecific individual odors are then closely associated with the nest and the colony as a whole.

The memory of individual identities is advantageous when contacts are repeated among a small number of individuals. This is obvious in hierarchical conflicts (D'Ettorre \& Heinze, 2005; Dreier et al., 2007; Tibbetts, 2002). This advantage is not yet known in heterospecific encounters, but our study reveals substantive cognitive and mnemonic abilities in a biological model, the ant, until then largely ignored in such research. We show that adult workers are able to learn and maintain in memory complex chemical information for heterospecific individual odors for at least $30 \mathrm{~min}$, without reinforcement, in a neutral context, in which the ant experiences no hierarchical conflict over food or colony defense. Moreover, this information is available for a discrimination task between two very close odors after a relatively long duration of at least $30 \mathrm{~min}$, but less than $60 \mathrm{~min}$.

Neurological processes linked to our observations are still unknown. Understanding them may allow us to conduct a comparative study with models such as honeybees and fruit flies, which are relatively well known neuroanatomically, molecularly, and genetically (see Davis, 2005, on Drosophila).

\section{AUTHOR NOTE}

We are grateful to A. Lenoir, P. Gouat, and R. Fénéron for helpful discussion and suggestions; J.-L. Durand for helping in statistics analysis; K. Hollis and three anonymous referees for comments; M. C. Malherbe for rearing the ants; and L. Baltenneck for revising the first version of this article in English. E.F. is now affiliated with UMR CNRS 5558-LBBE, Université Claude Bernard-Lyon 1. Correspondence concerning this article should be addressed to E. Nowbahari, Laboratoire d'Ethologie Ex- 
périmentale et Comparée UMR CNRS 7153, Université Paris 13, 99 Avenue J.-B. Clément, 93430 Villetaneuse, France (e-mail: elise.nowbahari@, leec.univ-paris13.fr).

\section{REFERENCES}

Ataya, H., \& Lenoir, A. (1984). Le comportement nécrophorique chez la fourmi Lasius niger L. [Necrophoric behavior of Lasius niger L.]. Insectes Sociaux, 31, 20-33.

BREed, M. D., \& BenNetT, B. (1987). Kin recognition in highly eusocial insects. In D. J. C. Fletcher \& C. D. Michener (Eds.), Kin recognition in animals (pp. 243-286). New York: Wiley.

Chameron, S., Schatz, B., Pastergue-Ruiz, I., Beugnon, G., \& Collett, T. S. (1998). The learning of a sequence of visual patterns by the ant Cataglyphis cursor. Proceedings of the Royal Society of London B, 265, 2309-2313.

Cheng, K., \& Wignall, A. E. (2006). Honeybees (Apis mellifera) holding on to memories: Response competition causes retroactive interference effects. Animal Cognition, 9, 141-150.

Crozier, R. H. (1987). Genetic aspects of kin recognition: Concepts, models, and synthesis. In D. J. C. Fletcher \& C. D. Michener (Eds.), Kin recognition in animals (pp. 55-73). New York: Wiley.

Crozier, R. H., \& Pamilo, P. (1996). Evolution of social insect colonies: Sex allocation and kin selection. Oxford: Oxford University Press.

DAvis, R. L. (2005). Olfactory memory formation in Drosophila: From molecular to systems neuroscience. Annual Review of Neuroscience, 28, 275-302

Delattre, O., \& Nowbahari, E. (2007). [Individual discrimination among sisters in the ant Cataglyphis cursor]. Unpublished raw data.

D'Ettorre, P., \& Heinze, J. (2005). Individual recognition in ant queens. Current Biology, 15, 2170-2174.

Dreier, S., van Zweden, J. S., \& D'ETtorre, P. (2007). Long-term memory of individual identity in ant queens. Biology Letters, 3, 459-462.

Dupuy, F., Sandoz, J.-C., Giurfa, M., \& Josens, R. (2006). Individual olfactory learning in Camponotus ants. Animal Behaviour, $\mathbf{7 2}$, 1081-1091.

ERRARD, C. (1994). Long-term memory involved in nestmate recognition in ants. Animal Behaviour, 48, 263-271.

Errard, C., Hefetz, A., \& Jaisson, P. (2006). Social discrimination tuning in ants: Template formation and chemical similarity. Behavioral Ecology \& Sociobiology, 59, 353-363.

Hammer, M., \& Menzel, R. (1995). Learning and memory in the honeybee. Journal of Neuroscience, 15, 1617-1630.

HorRIDGE, A. (2006). Visual discriminations of spokes, sectors, and circles by the honeybee (Apis mellifera). Journal of Insect Physiology, 52, 984-1003.

ICHIKAWA, N., \& SASAKI, M. (2003). Importance of social stimuli for the development of learning capability in honeybees. Applied Entomology \& Zoology, 38, 203-209.

IsINGRINI, M., LeNOIR, A., \& JAISSON, P. (1985). Preimaginal learning as a basis of colony-brood recognition in the ant Cataglyphis cursor. Proceedings of the National Academy of Sciences, 82, 8545-8547.

JAISSON, P. (1974). L'imprégnation dans l'ontogenèse des comportements de soins aux cocons chez la jeune fourmi rousse (Formica polyctena Först). Behaviour, 52, 1-37.

Lahav, S., Soroker, V., Hefetz, A., \& Vander Meer, R. K. (1999). Direct behavioral evidence for hydrocarbons as ant recognition discriminators. Naturwissenschaften, 86, 246-249.
Lenoir, A., Fresneau, D., Errard, C., \& Hefetz, A. (1999). Individuality and colonial identity in ants: The emergence of the social representation concept. In C. Detrain, J. L. Deneubourg, \& J. M. Pasteels (Eds.), Information processing in social insects (pp. 219-223). Basel: Birkhäuser.

Macquart, D., \& Beugnon, G. (2004). L'apprentissage de routes familières chez la fourmi néotropicale Gigantiops destructor. Actes des Colloques Insectes Sociaux, 16, 70-74.

Matsumoto, Y., \& Mizunami, M. (2000). Olfactory learning in the cricket Gryllus bimaculatus. Journal of Experimental Biology, 203, 2581-2588.

Matsumoto, Y., \& Mizunami, M. (2005). Formation of long-term olfactory memory in the cricket Gryllus bimaculatus. Chemical Senses, 30, 1299-1300.

Nielsen, J., Boomsma, J. J., Oldham, N. J., Petersen, H. C., \& MorGAN, E. D. (1999). Colony-level and season-specific variation in cuticular hydrocarbon profiles of individual workers in the ant Formica truncorum. Insectes Sociaux, 46, 58-65.

NOWBAHARI, E. (2007). Learning of colonial odor in the ant Cataglyphis niger (Hymenoptera; Formicidae). Learning \& Behavior, 35, 87-94.

Provost, E., Bonavita-Cougourdan, A., \& Rivière, G. (1994). Plasticité du profil cuticulaire spécifique des hydrocarbures chez les fourmis: Facteurs physiologiques et environnementaux. Actes des Colloques Insectes Sociaux, 9, 1-10.

SaKuRA, M., \& Mizunami, M. (2001). Olfactory learning and memory in the cockroach Periplaneta americana. Zoological Science, 18, 21-28.

SaKura, M., OKada, R., \& Mizunami, M. (2002). Olfactory discrimination of structurally similar alcohols by cockroaches. Journal of Comparative Physiology, 188, 787-797.

TiвbETTs, E. A. (2002). Visual signals of individual identity in the wasp Polistes fuscatus. Proceedings of the Royal Society of London B, 269, 1423-1428.

Todrank, J., \& Heth, G. (2003). Odor-genes covariance and genetic relatedness assessments: Rethinking odor-based "recognition" mechanisms in rodents. In P. J. B. Slater, J. S. Rosenblatt, C. T. Snowdon, \& T. J. Roper (Eds.), Advances in the study of behavior (Vol. 32, pp. 77-130). San Diego: Academic Press.

Vander Meer, R. K., \& Morel, L. (1998). Nestmate recognition in ants. In R. K. Vander Meer, M. D. Breed, M. Winston, \& C. Espelie (Eds.), Pheromone communication in social insects: Ants, wasps, bees and termites (pp. 79-103). Boulder, CO: Westview.

Vander Meer, R. K., Saliwanchik, D., \& Lavine, B. (1989). Temporal changes in colony cuticular hydrocarbon patterns of Solenopsis invicta: Implications for nestmate recognition. Journal of Chemical Ecology, 15, 2115-2125.

Watanabe, H., Kobayashi, Y., Sakura, M., Matsumoto, Y., \& MizuNAMI, M. (2003). Classical olfactory conditioning in the cockroach Periplaneta americana. Zoological Science, 20, 1447-1454.

Wilson, E. O., Durlach, N. I., \& Roth, L. M. (1958). Chemical releasers of necrophoric behavior in ants. Psyche, 65, 108-114.

Zhang, S., Mizutani, A., \& Srinivasan, M. V. (2000). Maze navigation by honeybees: Learning path regularity. Learning \& Memory, 7, 363-374.

(Manuscript received April 8, 2008, revision accepted for publication April 24, 2008.) 